Paris, France; ${ }^{3}$ Hôpital Cochin - APHP, Rheumatology, Paris, France; ${ }^{4}$ Hôpital Sud-Francillien, Pharmacy, Corbeil-Essonnes, France; ${ }^{5}$ Hôpital Necker - Enfants Malades, Pharmacy, Paris, France; ${ }^{6}$ Hôpital Cochin - APHP, Risk and quality management, Paris, France

Background: In chronic rheumatic diseases, non-adherence to treatment is associated with a progression of disease and an increased morbidity (1). In spondyloarthritis (SpA), improving patients' knowledge on their subcutaneous biologic disease-modifying antirheumatic drugs (bDMARDs) is a key factor to enhance medication adherence (2). The patient information has to ensure the acquisition of safety skills regarding their treatment management.

Objectives: To evaluate the impact of a pharmacist's educational interview on knowledge and therapeutic adherence of subcutaneous bDMARDs in patients with SpA.

Methods: Population and study design: consecutive adult patients with well-controlled axial SpA, stable on subcutaneous bDMARDs were enrolled in a randomized, controlled, single-center, open-label, 6-months trial. Intervention: A pharmacist's educational interview provided information on bDMARDs management at baseline in the intervention group (IG) and at month 6 (M6) in the control group (CG). A booklet containing essential information was given to the patient. Intervention allocation: After written consent, the study treatment was randomly allocated via a computer program by simple randomization, with an allocation ratio of 1:1. Outcome measures: The change of a weighted knowledge score $(0$ -100) concerning the bDMARDs management and the change in the Medication Possession Ratio (MPR) at M6 were primary outcomes. The changes in disease activity (BASDAI) and patients' satisfaction regarding the pharmacists' interview were secondary outcomes. Statistics: Changes in knowledge score, MPR and BASDAI were compared between the two groups using the T-Student test. Statistical analysis was performed in intention-to-treat. Missing data was handled with multiple imputations.

Results: Patients' characteristics at baseline were comparable among the 89 included patients (46 in IG, 43 in CG). The means \pm SD of the knowledge score were $75.3 \pm 14.2$ versus $73.0 \pm 13.2$ and $86.3 \pm 12.6$ versus $76.0 \pm 14.1$ in the IG versus $C G$ at baseline and at M6, respectively. The patient's knowledge score improved at a greater magnitude in the IG $(+11.0 \pm 11.5$ versus $+3.0 \pm 10.6$ in the $I G$ versus the $C G$ respectively, $p<0.0001)$. The MPR at baseline were very high in both groups $(92.9 \pm 14.6 \%$ versus $96.6 \pm 15.6 \%$ in the $I G$ versus the $C G$, respectively). There was a trend in a better adherence $(+2.2 \pm 13.9$ versus $-0.6 \pm 18.9$ in the IG versus the CG in the MPR score respectively, $p=0,691$ ). The disease activity (changes in BASDAI) remained stable during the study in both groups. All the patients were mostly or totally satisfied by the pharmacists' interview.

Conclusion: Pharmacists' educational interview on subcutaneous bDMARDs is effective in improving the knowledge of patients with SpA on their treatment. Regarding therapeutic adherence, a trend in favor of an improvement was observed in the intervention group but did not reach the statistically significance. Nevertheless, the results observed in this study are an argument to propose to include the pharmacists in the multidisciplinary team in charge of the management of patients with SpA.

References:

[1] Bluett J, Morgan C, Thurston L et al. Impact of inadequate adherence on response to subcutaneously administered anti-tumour necrosis factor drugs: results from the biologics in rheumatoid. Rheumatology. 2015;54(3):494-9.

[2] Gossec L, Molto A, Romand X et al. Recommendations for the assessment and optimization of adherence to disease-modifying drugs in chronic inflammatory rheumatic diseases: A process based on literature reviews and expert consensus. Joint Bone Spine. 2019;86(1):13-9.

Disclosure of Interests: Loriane Gutermann: None declared, Sophie Dumas: None declared, Clementina López-Medina: None declared, Léa Boissinot: None declared, Camille Cotteret: None declared, Valérie Perut: None declared, Anna Moltó Grant/research support from: Pfizer, UCB, Consultant of: Abbvie, BMS, MSD, Novartis, Pfizer, UCB, Ornella Conort: None declared, Maxime Dougados Grant/research support from: AbbVie, Eli Lilly, Merck, Novartis, Pfizer and UCB Pharma, Consultant of: AbbVie, Eli Lilly, Merck, Novartis, Pfizer and UCB Pharma, Speakers bureau: AbbVie, Eli Lilly, Merck, Novartis, Pfizer and UCB Pharma DOI: 10.1136/annrheumdis-2020-eular.430

\section{FRI0623-HPR HEALTHCARE RESOURCE USE IN PATIENTS WITH TRAPEZIOMETACARPAL OSTEOARTHRITIS}

T. Hamasaki ${ }^{1,2,3}$, P. Harris ${ }^{1,2,4}$, N. Bureau ${ }^{1,2,3}$, N. Gaudreault ${ }^{5,6}$, N. Patenaude ${ }^{5,6,7}$, M. Choinière ${ }^{2,3} \cdot{ }^{1}$ CHUM Hospital (Université de Montréal), Montréal, Canada; ${ }^{2}$ Université de Montréal, Montréal, Canada; ${ }^{3}$ Centre de recherche du CHUM (CRCHUM), Montreal, Canada; ${ }^{4}$ Research Centre of the CHUM, Montreal, Canada; ${ }^{5}$ Université de Sherbrooke, Sherbrooke, Canada; ${ }^{6}$ Centre de recherche du CHUS (CRCHUS), Sherbrooke, Canada; ${ }^{7} \mathrm{CHUS} \mathrm{Hospital} \mathrm{(Université} \mathrm{de}$ Sherbrooke), Sherbrooke, Canada
Background: Trapeziometacarpal osteoarthritis (TMO) is one of the most debilitating forms of hand osteoarthritis (OA). According to the recent EULAR guidelines ${ }^{1,2}$ and a systematic review, ${ }^{3}$ the efficacy of topical/oral non-steroid anti-inflammatory drugs (NSAIDs), orthoses, hand exercises, and psychosocial interventions for hand OA or TMO are supported by scientific evidence. Cortisone injections and acetaminophen are generally not recommended. ${ }^{1{ }^{3}}$ Besides, TMO management is suboptimal: only $21 \%$ of patients receive rehabilitative interventions prior to referral to hand surgeons. ${ }^{4}$

Objectives: We aimed at documenting the types of treatment TMO patients employ and their healthcare resource use.

Methods: A total of $227 \mathrm{TMO}$ patients recruited from 16 healthcare institutions completed a questionnaire about 1) received interventions, 2) analgesic strategies, and 3) healthcare professional consultations.

Results: Acetaminophen (64.3\% of the participants), oral NSAIDs (31.7\%), topical NSAIDs $(11.9 \%)$, and nutraceuticals $(7.9 \%)$ were the most commonly used medications. More than $70 \%$ of the participants reported having received cortisone injection(s) $(72.5 \%)$ and orthosis $(75.7 \%)$. More than half employed hand exercises massage and heat/cold application. Relaxation/respiration, meditation, distraction, assistive devices, and joint protection principles were used by smaller percentages of participants (13.0-30.9\%). Patients with TMO reported having consulted various types of healthcare professionals: family physicians, plastic/orthopaedic surgeons, radiologists-interventionists, rheumatologists, physiatrists, occupational/physical therapists, osteopaths, chiropractors, pharmacists, and acupuncturists. Only $4.8 \%$ of the participants reported having received psychosocial interventions.

Conclusion: TMO patients use numerous types of modalities to relieve their pain. Provision of evidence-based interventions tailored to their needs is clearly needed.

References:

[1] Kloppenburg M, Kroon FP, Blanco FJ, et al. 2018 update of the EULAR recommendations for the management of hand osteoarthritis. Annals of the rheumatic diseases. 2019;78:16-24.

[2] Geenen R, Overman CL, Christensen R, et al. EULAR recommendations for the health professional's approach to pain management in inflammatory arthritis and osteoarthritis. Annals of the rheumatic diseases. 2018;77:797-807.

[3] Hamasaki T, Laprise S, Harris PG, et al. Efficacy of non-surgical interventions for trapeziometacarpal (thumb base) osteoarthritis: A systematic review. Arthritis Care \& Research. 2019; In press (https://doi.org/10.1002/acr.24084).

[4] Gravås EMH, Tveter AT, Nossum R, et al. Non-pharmacological treatment gap preceding surgical consultation in thumb carpometacarpal osteoarthritis - a cross-sectional study. BMC musculoskeletal disorders. 2019;20:180-180. Acknowledgments: This study was supported by a discretionary fund of the Centre de recherche du CHUM (CRCHUM) to Choinière and from the Multidisciplinary Council of the CHUM. Hamasaki was supported by a Doctoral training award of the Fonds de recherche du Québec-Santé, a doctoral scholarship from the CHUM Foundation to Harris (Hand Surgery Branch) and from Choinière's internal funds of the CRCHUM.

Disclosure of Interests: None declared

DOI: 10.1136/annrheumdis-2020-eular.556

\section{FRI0624-HPR A SYSTEMATIC REVIEW OF JOB LOSS PREVENTION INTERVENTIONS FOR PERSONS WITH INFLAMMATORY ARTHRITIS.}

C. M. T. Madsen ${ }^{1,2}$, S. Kjaer ${ }^{3}$, J. Primdahl1,2,4 J. R. Christensen ${ }^{5}$, C. Von Bülow ${ }^{3,6}$ ${ }^{1}$ Danish Hospital for Rheumatic Diseases, Sønderborg, Denmark; ${ }^{2}$ Department of Regional Health Research, University of Southern Denmark, Odense, Denmark; ${ }^{3}$ Research Initiative for Activity Studies and Occupational Therapy, Research unit for General Practice, Department of Public Health, University of Southern Denmark, Odense, Denmark; ${ }^{4}$ Hospital of Southern Jutland, University Hospital of Southern Denmark, Aabenraa, Denmark; ${ }^{5}$ Research unit for General Practice, Department of Public Health, University of Southern Denmark, Odense, Denmark; ${ }^{6}$ The Parker Institute, Copenhagen University Hospital, Bispebjerg and Frederiksberg, Denmark

Background: Persons with inflammatory arthritis (IA) have a higher level of absenteeism from work than those without IA and up to $20-30 \%$ become permanently work-disabled during the first years after being diagnosed with IA. Despite developments of new pharmacological and surgical treatments, people with IA still report reduced work ability. It is therefore relevant to offer effective interventions designed to prevent job loss and improve work function (i.e. job loss prevention interventions) to support people with IA to stay connected to the labour market. Initial effects of job loss prevention interventions have been established in a Cochrane review by Hoving et al. 2014 (1), but as only three randomized controlled trials (RCT) were identified, it seems relevant to investigate if new evidence has emerged. 